\title{
Agôn
}

Revue des arts de la scène

Critiques | Saison 2011-2012

\section{Le Corps qui iMAGEinait plus vite que son ombre}

Un Mage en été d'Olivier Cadiot, mis en scène par Ludovic Lagarde

\section{Marion Siéfert}

\section{(2) OpenEdition}

Journals

\section{Édition électronique}

URL : http://journals.openedition.org/agon/2235

DOI : 10.4000/agon.2235

ISSN : 1961-8581

Éditeur

Association Agôn

Référence électronique

Marion Siéfert, "Le Corps qui iMAGEinait plus vite que son ombre », Agôn [En ligne], Critiques, mis en ligne le 13 mai 2012, consulté le 23 septembre 2020. URL : http://journals.openedition.org/agon/2235 ; DOI : https://doi.org/10.4000/agon.2235

Ce document a été généré automatiquement le 23 septembre 2020

Association Agôn et les auteurs des articles 


\section{Le Corps qui iMAGEinait plus vite que son ombre}

Un Mage en été d'Olivier Cadiot, mis en scène par Ludovic Lagarde

\section{Marion Siéfert}

1 Que voyons-nous dans Un Mage en été ? Presque rien. Qu'imaginons-nous ? Presque tout. Et c'est la voix d'un mage qui, dans l'esprit des spectateurs, fait jaillir des visions. Dernière création du trio Cadiot - Lagarde - Poitrenaux, ce monologue nous embarque, au détour de la balade, vers des territoires jusqu'alors inconnus au théâtre, empruntant au cinéma ce que le corps théâtral ne peut produire : des images et des sauts spatiauxtemporels. Oscillant entre une aspiration à tout imaginer ou à n'être rien, le spectacle nous met au défi d'imaginer ce que peut un corps. 
Un Mage en été d'Olivier Cadiot, mis en scène par Ludovic Lagarde

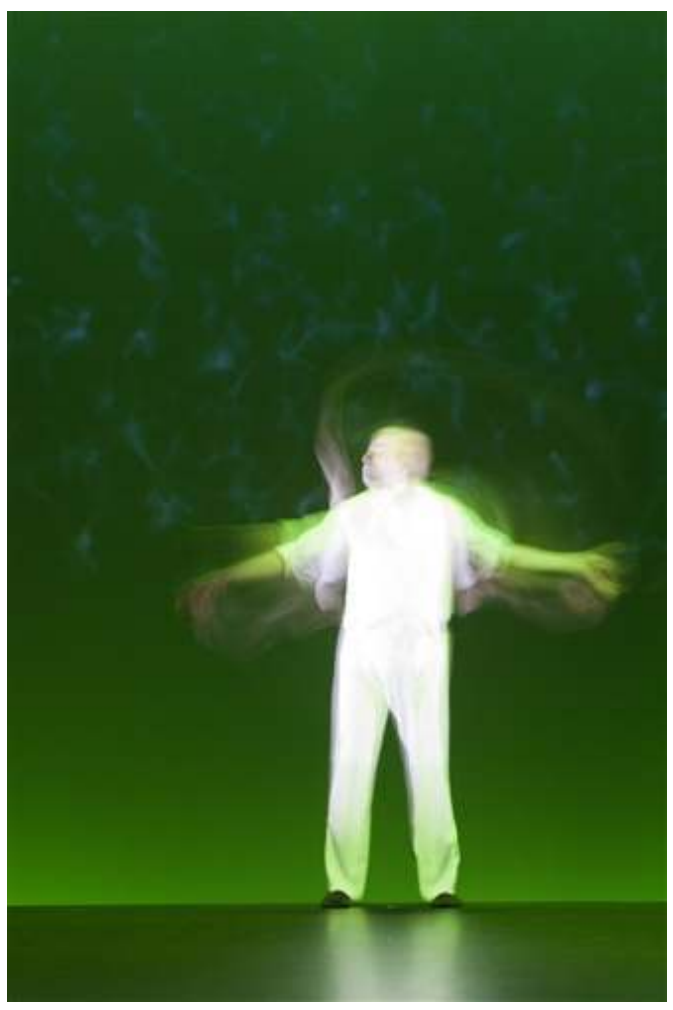

Laurent Poitrenaux

(c) Marthe Lemelle

2 D'emblée, Un Mage en été semble se passer du corps, de la vision : injonction au spectateur à fermer les yeux, noir introductif. Et lorsque Laurent Poitrenaux imite la voix de Jean-Luc Godard pour faire entendre un défi esthétique, faire un film les yeux fermés, la représentation théâtrale tourne à l'exercice périlleux. Ne plus rien voir, c'est ne plus rien représenter : fixer des vertiges, en somme. Si l'on se souvient des interventions de Godard à la télévision, on comprend alors mieux ce que signifie regarder un film les yeux fermés. C'est se concentrer sur la bande-son. Accepter le pacte que noue ce narrateur-mage avec ses spectateurs implique que l'on ferme les yeux et laisse se dérouler le film projeté sur ses tympans. Car il n'y a pas grand chose à voir sur scène : quelques changements de lumière, des images lointaines, un homme, toujours le même, en costume d'été. Les images surgissent des mots, d'une inflexion de la voix, du bruit d'une rivière... Et voilà le public devenu spectateur-monteur, piochant dans la matière abondante et fugace des évocations du mage pour créer son propre film. Navigant à travers des médias divers, la narration enfile les visions : on entre dans une photographie et se retrouve dans une rivière, au plus près des sensations d'une femme, belle, sensuelle, qui se rafraîchit dans les eaux scintillantes du cours d'eau. Puis, on évoque Theodor W. Adorno et assiste à une scène dans laquelle Friedrich Nietzsche jouerait au golf. Avant de revenir à la femme, toujours trempée dans la fraîcheur de l'eau. Les images défilent comme sur un écran d'ipad, d'où surgissent d'inépuisables bases d'archives, sonores et visuelles. Parfois, le film se rembobine et le mage monte sous nos yeux une bande-son différente, piochant dans de vastes bibliothèques numériques, semblant contenir tous les trésors du monde. Nous sommes dans l'atelier d'un créateur d'imaginaire. De mage à image, la transition est rapide. 
Ce voyage dans l'atelier mental du mage-Poitrenaux se passe de la représentation, d'une incarnation sur le plateau des images évoquées. La continuité temporelle et spatiale de la scène de théâtre est donc à mille lieues, puisque ce personnage indéfinissable qui, au cours du spectacle trouve son identité de mage, peut convoquer toutes les images possibles et imaginables. La référence esthétique centrale est donc celle du cinéma, plus plastique que le théâtre, qui se passe des corps, coupe dans le réel et monte, afin de faire se rencontrer des images que l'on ne pourrait jamais voir côte-àcôte dans la réalité. À la continuité, Un Mage en été préfère le saut, comme si l'on franchissait des territoires aux frontières extrêmement plastiques et élastiques. Le génie de la mise en scène de Ludovic Lagarde est de ne pas avoir cherché à accessoiriser le texte, à faire de la scène un atelier de savant pratiquant des sciences occultes, avec différents objets qui permettraient de contextualiser et d'illustrer la fonction de ce mage moderne. Au contraire, la scénographie d'Antoine Vasseur crée un cube noir, c'est-à-dire un cadre dans le théâtre, au sol légèrement bombé, permettant ainsi à Laurent Poitrenaux de changer d'échelle. Le fond de la scène est occupé par un cyclorama, décor invisible par excellence, sur lequel surgissent et s'évanouissent des images, sans support réel. Habillée parfois de vert fluo, cette boîte noire devient ce lieu vierge, dans lequel est projeté Poitrenaux. Du cinéma, la mise en scène a retenu non seulement le montage, mais aussi le travail minutieux du son. Encerclé d'enceintes, le public évolue dans un espace sonore extrêmement riche, guidé par la voix du mage, amplifiée au micro HF. Les intonations de Poitrenaux sont donc travaillées, modifiées, amplifiées, grâce aux techniques sonores de l'IRCAM, menées par David Bichindaritz en partenariat avec Grégory Beller. La voix de Poitrenaux est à la mesure de ses visions ; aux nuances infinies, elle caresse des vertiges et fixe des illuminations. Les sorties de micro, réparties tout autour de la salle, permettent de créer un espace sonore commun et de jouer avec les changements de lieu, les déplacements du mage. On pourrait conclure promptement à un théâtre désincarné, effaçant la scène dans un noir qui ressemble trop à celui d'une salle de cinéma. Mais, dans Un Mage en été, le corps resurgit là où on ne l'attend pas, aux détours du texte et dans une mise en question profonde du mage de sa propre identité.

4 Le mage possède en effet tous les attributs du personnage fétiche d'olivier Cadiot : Robinson. " Héros sans expérience ", c'est-à-dire terre vierge par excellence, sa seule particularité est de "repartir de zéro à chaque fois ". Dépourvu des attributs encombrants de la personnalité bien marquée des personnages de roman, Robinson est le personnage de tous les possibles, des métamorphoses et des appropriations. Or, Olivier Cadiot le soumet dans Un Mage en été à une autocritique sévère. Dès lors, ce corps n'est plus désincarné mais subit un véritable « torture-test ", lorsque ses visions suscitent une souffrance. Un corps assailli d'images, c'est le triste lot du Robinson moderne, pour qui les moyens fabuleux de la technique sont désormais les vecteurs d'une imagination compulsive. Si l'imagination, la virtualité se trouvent être des fardeaux, le repos se trouve paradoxalement dans le corps dont les sensations se précisent au photon près. Alors qu'il imagine plus vite que son ombre, le corps du mage met au défi l'imagination, la dépasse : «vous n'imaginez pas ce que peut un corps ». C'est dans la technologie la plus pointue, celle des pixels, des écrans ipad et des bases de données virtuelles, que ce corps prend forme, pour se métamorphoser : " Je me compose, je me décompose ». Sur scène, le corps de l'acteur Poitrenaux est façonné par un travail de la détente, élaboré avec la chorégraphe Stéfany Ganachaud. Survolant le corps-machine cartésien, la langue de Cadiot prend matière dans un corps magique, 
disposant de super-pouvoirs, ceux de la technologie, et dont la langue agit comme autant de formules magiques.

5 Animé par une véritable tension dramatique, Un Mage en été ne se résorbe pas dans une écriture du thème et de la variation, tournant autour de la figure du mage, mais se livre au contraire à une véritable introspection poïétique, à une descente dans l'atelier de l'écrivain, territoire vierge indéfini ou écran assailli de visions. Comment caresser des images? L'émotion qui naît de ce spectacle n'est pas provoquée par l'empathie que l'on éprouve vis-à-vis d'un personnage attachant, mais plutôt par la précision infinie des sensations, des images évoquées, dévoilées dans l'écrin précieux de leur particularité par un personnage insaisissable. "Produire du lyrisme par d'autres moyens qu'en convoquant des émotions ", une exigence qu'Olivier Cadiot s'est imposée dès ses débuts en littérature. Remercions-le car il est rare que la précision suscite l'émotion, que le spectaculaire ou l'identification ne soient pas les seuls convoqués pour émouvoir. le 10 mai 2012 à L'Équinoxe, Scène nationale de Châteauroux

7 le 15 mai 2012 à La Passerelle, Scène nationale de Saint-Brieuc

8 du 24 mai au 3 juin 2012 au Théâtre du Rond-Point, Paris.

INDEX

Mots-clés : Cadiot (Olivier), Lagarde (Ludovic), Un Mage en été 